\title{
On the Exponential Decay of Solutions for Some Kirchhoff-Type Modelling Equations with Strong Dissipation
}

\author{
Yaojun Ye \\ Department of Mathematics and Information Science, Zhejiang University of Science and Technology, \\ Hangzhou, China \\ E-mail: yeyaojun2002@yahoo.com.cn \\ Received August 24, 2010; revised October 19, 2010; accepted October 23, 2010
}

\begin{abstract}
This paper deals with the initial boundary value problem for a class of nonlinear Kirchhoff-type equations with strong dissipative and source terms $u_{t t}-\varphi\left(\|\nabla u\|_{2}^{2}\right) \Delta u-a \Delta u_{t}=b|u|^{\beta-2} u, x \in \Omega, t>0$ in a bounded domain, where $a, b>0$ and $\beta>2$ are constants. We obtain the global existence of solutions by constructing a stable set in $H_{0}^{1}(\Omega)$ and show the energy exponential decay estimate by applying a lemma of V. Komornik.
\end{abstract}

Keywords: Kirchhoff-type Equation; Initial Boundary Value Problem; Stable Set; Exponential Decay Estimate

\section{Introduction}

Let $\Omega$ be a bounded domain in $R^{n}$ with smooth boundary $\partial \Omega$. In this paper, we investigate the existence and the energy exponential decay estimate of global solutions for the initial boundary value problem of the following Kirchhoff-type equation with strong dissipative and source terms in a bounded domain

$$
\begin{gathered}
u_{t t}-\varphi\left(\|\nabla u\|_{2}^{2}\right) \Delta u-a \Delta u_{t}=b|u|^{\beta-2} u, \quad x \in \Omega, t>0, \\
u(x, 0)=u_{0}(x), \quad u_{t}(x, 0)=u_{1}(x), \quad x \in \Omega, \\
u(x, t)=0, \quad x \in \partial \Omega, \quad t \geq 0,
\end{gathered}
$$

where $a, b>0$ and $\beta>2$ are constants, $\varphi(s)$ is a $C^{1}$-class function on $[0,+\infty)$ satisfying

$$
\varphi(s) \geq m_{0}, s \varphi(s) \geq \int_{0}^{s} \varphi(\theta) d \theta, \forall s \in[0,+\infty)
$$

with $m_{0} \geq 1$ constant.

When $n=1$, the equation (1.1) describes a small amplitude vibration of an elastic string ([1]). The original equation is

$$
\rho h \frac{\partial^{2} u}{\partial t^{2}}+\tau \frac{\partial u}{\partial t}=\left(P_{0}+\frac{E h}{2 L} \int_{0}^{L}\left|\frac{\partial u}{\partial x}\right| d s\right) \frac{\partial^{2} u}{\partial x^{2}}+f
$$

where $0 \leq x \leq L$ and $t>0, u(x, t)$ is the lateral displacement at the space coordinate $x$ and the time $t, \rho$ is the mass density, $h$ is the cross-section area, $L$ is the length, $P_{0}$ is the initial axial tension, $\tau$ is the resistance modulus, $E$ is the Young modulus and $f$ is the external force.

Many authors have studied the existence and uniqueness of solutions of (1.1)-(1.3) by using various methods. When $a, b>0$, and $\varphi(s)=s^{r}, r \geq 1$, K. Nishihara and Y. Yamada [2] have proved the existence and the polynomial decay of global solution under the assumptions that the initial data $u_{0}$ and $u_{1}$ are sufficiently small and $u_{0} \neq 0$. However, the method in [2] can not be applied directly to the case that the equations have the blow-up term $|u|^{\beta-2} u$. M. Aassila and A. Benaissa [3] extend the global existence part of [2] to the case where $\varphi(s)>0$ with $\varphi\left(\left\|\nabla u_{0}\right\|^{2}\right) \neq 0$ and the nonlinear dissipative term $\left|u_{t}\right|^{\alpha-2} u_{t}$. K. Ono and K. Nishihara [4] have proved the global existence and decay structure of solutions of (1.1)-(1.3) without small condition of data using Galerkin method. K. Ono [5] has obtained the global existence of solutions for the problem (1.1)-(1.3) with dissipative term $u_{t}$ instead of $\Delta u_{t}$.

In the case $a=0$, for large $\beta$ and $\varphi(s) \geq r>0, \mathrm{P}$. 
D. Ancona and S. Spagnolo [6] proved that if $u_{0}, u_{1} \in C_{0}^{\infty}\left(R^{n}\right)$ are small, then problem (1.1)-(1.3) has a global solution. When $\varphi(s) \geq 0$, M. Ghisi and M. Gobbino [7] proved the existence and uniqueness of a global solution $u(x, t)$ of (1.1)-(1.3) for small initial data

$$
\left(u_{0}, u_{1}\right) \in\left(H_{0}^{1}(\Omega) \cap H^{2}(\Omega)\right) \times H_{0}^{1}(\Omega)
$$

with $m\left(\left\|\nabla u_{0}\right\|^{2}\right) \neq 0$

and the asymptotic behavior

$$
\left(u(t), u_{t}(t), u_{t t}(t)\right) \rightarrow\left(u_{\infty}, 0,0\right)
$$

in $\left(u_{0}, u_{1}\right) \in\left(H_{0}^{1}(\Omega) \cap H^{2}(\Omega)\right) \times H_{0}^{1}(\Omega) \times L^{2}(\Omega)$

as $t \rightarrow+\infty$, where either $u_{\infty}=0$ or $\varphi\left(\left\|\nabla u_{\infty}\right\|^{2}\right)=0$.

The case $\varphi(s) \geq r>0$ has been considered by $\mathrm{M}$. Hosoya and Y. Yamada [8] under the following condition:

$$
0 \leq \beta<\frac{2}{n-4}, n \geq 5 ; \quad 0 \leq \beta<+\infty, n \leq 4 .
$$

They proved that, if the initial data are small enough, the problem (1.1)-(1.3) has a global solution which decays exponentially as $t \rightarrow+\infty$.

In this paper, we prove the global existence for the problem (1.1)-(1.3) by applying the potential well theory introduced by D. H. Sattinger [9] and L. Payne and D. H. Sattinger [10]. Meanwhile, we obtain the exponential decay estimate of global solutions by using the different method from paper [8].

We adopt the usual notation and convention. Let $H^{m}$ denote the Sobolev space with the norm

$$
\|u\|_{H^{m}(\Omega)}=\left(\sum_{|\alpha| \leq m}\left\|D^{\alpha} u\right\|_{L^{2}(\Omega)}^{2}\right),
$$

$H_{0}^{m}(\Omega)$ denotes the closure in $H^{m}$ of $C_{0}^{\infty}(\Omega)$. For simplicity of notations, hereafter we denote by $\|\cdot\|_{p}$ the Lebesgue space $L^{p}(\Omega)$ norm, $\|\cdot\|$ denotes $L^{2}(\Omega)$ norm and we write equivalent norm $\|\nabla \cdot\|$ instead of $H_{0}^{1}(\Omega)$ norm $\|\cdot\|_{H_{0}^{1}(\Omega)}$. Moreover, $M$ denotes various positive constants depending on the known constants and it may be different at each appearance.

\section{Preliminary}

In order to state and prove our main results, we first define the following functionals

$$
K(u)=m_{0}\|\nabla u\|^{2}-b\|u\|_{\beta}^{\beta}, \quad J(u)=\frac{m_{0}}{2}\|\nabla u\|^{2}-\frac{b}{\beta}\|u\|_{\beta}^{\beta}
$$

for $u \in H_{0}^{1}(\Omega)$. Then we define the stable set $S$ by

$$
S=\left\{u \in H_{0}^{1}(\Omega), K(u)>0, J(u)<d\right\} \cup\{0\},
$$

where

$$
d=\inf \left\{\sup _{\lambda>0} J(\lambda u), u \in H_{0}^{1}(\Omega) /\{0\}\right\} .
$$

We denote the total energy functional associated with (1.1)-(1.3) by

$$
E(t)=\frac{1}{2}\left\|u_{t}\right\|_{2}^{2}+\frac{1}{2} \int_{0}^{\|\nabla u\|^{2}} \varphi(s) d s-\frac{b}{\beta}\|u\|_{\beta}^{\beta}
$$

for $u \in H_{0}^{1}(\Omega), t \geq 0$, and

$$
E(0)=\frac{1}{2}\left\|u_{1}\right\|_{2}^{2}+\frac{1}{2} \int_{0}^{\left\|\nabla u_{0}\right\|^{2}} \varphi(s) d s-\frac{b}{\beta}\left\|u_{0}\right\|_{\beta}^{\beta}
$$

is the total energy of the initial data.

Lemma 2.1 Let $q$ be a number with $2 \leq q<+\infty$, $n \leq 2$ and $2 \leq q \leq \frac{2 n}{n-2}, n>2$. Then there exists a constant $C$ depending on $\Omega$ and $q$ such that

$$
\|u\|_{q} \leq C\|u\|_{H_{0}^{1}(\Omega)}, \forall u \in H_{0}^{1}(\Omega) \text {. }
$$

Lemma 2.2 [11] Let $y(t): R^{+} \rightarrow R^{+}$be a nonincreasing function and assume that there is a constant $A>0$, such that

$$
\int_{s}^{+\infty} y(t) d t \leq A y(s), 0 \leq s<+\infty,
$$

then $y(t) \leq y(0) e^{1-\frac{t}{\lambda}}, \forall t \geq 0$.

We state a local existence result, which is known as a standard one.

Theorem 2.1 Suppose that $\beta$ satisfies

$$
2<\beta<+\infty, n \leq 2 ; 2<\beta \leq \frac{2 n}{n-2}, n>2 .
$$

If $\left(u_{0}, u_{1}\right) \in H_{0}^{1}(\Omega) \cap L^{2}(\Omega)$, then there exists $T>0$ such that the problem (1.1)-(1.3) has a unique local solution $u(t)$ in the class

$u \in C\left([0, T) ; H_{0}^{1}(\Omega)\right), \quad u_{t} \in C\left([0, T) ; L^{2}(\Omega)\right)$.

Lemma 2.3 Let $u(t, x)$ be a solutions of problem (1.1)-(1.3). Then $E(t)$ is a nonincreasing function for $t>0$ and

$$
\frac{d}{d t} E(t)=-a\left\|\nabla u_{t}(t)\right\| .
$$

Proof By multiplying equation (1.1) by $u_{t}$ and integrating over $\Omega$, we get

$$
\frac{d}{d t} E(t)=-a\left\|\nabla u_{t}(t)\right\| \leq 0 .
$$

Therefore, $E(t)$ is a nonincreasing function on $t$.

Lemma 2.4 Let $u \in H_{0}^{1}(\Omega)$, if (2.2) holds, then $d>0$.

Proof Since

$$
J(\lambda u)=\frac{m_{0} \lambda^{2}}{2}\|\nabla u\|^{2}-\frac{b \lambda^{\beta}}{\beta}\|u\|_{\beta}^{\beta},
$$


so, we get

$$
\frac{d}{d \lambda} J(\lambda u)=m_{0} \lambda\|\nabla u\|^{2}-b \lambda^{\beta-1}\|u\|_{\beta}^{\beta} .
$$

Let $\frac{d}{d \lambda} J(\lambda u)=0$, which implies that

$$
\lambda_{1}=\left(\frac{b}{m_{0}}\right)^{-\frac{1}{\beta-2}}\left(\frac{\|u\|_{\beta}^{\beta}}{\|\nabla u\|^{2}}\right)^{-\frac{1}{\beta-2}} .
$$

As $\lambda=\lambda_{1}$, an elementary calculation shows that

$$
\frac{d^{2}}{d \lambda^{2}} J(\lambda u)<0 \text {. }
$$

Hence, we have from Lemma 2.1 that

$$
\begin{aligned}
\sup _{\lambda \geq 0} J(\lambda u) & =J\left(\lambda_{1} u\right)=\frac{\beta-2}{2 \beta}\left(\frac{b^{2}}{m_{0}^{\beta}}\right)^{-\frac{1}{\beta-2}}\left(\frac{\|u\|_{\beta}}{\|\nabla u\|}\right)^{-\frac{2 \beta}{\beta-2}} \\
& \geq \frac{\beta-2}{2 \beta}\left(\frac{b^{2}}{m_{0}^{\beta}}\right)^{-\frac{1}{\beta-2}} C^{-\frac{2 \beta}{\beta-2}}>0 .
\end{aligned}
$$

we get from the definition of $d$ that $d>0$.

In order to prove the existence of global solutions for the problem (1.1)-(1.3), we need the following Lemma.

Lemma 2.5 Supposed that (2.2) hold, If $u_{0} \in S, u_{1} \in L^{2}(\Omega)$ and $E(0)<d$, then $u \in S$, for each $t \in[0, T)$.

Proof Assume that there exists a number $t^{*} \in[0, T)$. such that $u(t) \in S$ on $\left[0, t^{*}\right)$ and $u\left(t^{*}\right) \notin S$. Then, in virtue of the continuity of $u(t)$, we see $u\left(t^{*}\right) \in \partial S$. From the definition of $S$ and the continuity of $J(u(t))$ and $K(u(t))$ in $t$, we have either

$$
J\left(u\left(t^{*}\right)\right)=d \text { or } K\left(u\left(t^{*}\right)\right)=0 \text {. }
$$

It follows from (1.4) and (2.1) that

$$
\begin{aligned}
J\left(u\left(t^{*}\right)\right) & =\frac{m_{0}}{2}\left\|\nabla u\left(t^{*}\right)\right\|^{2}-\frac{b}{\beta}\left\|u\left(t^{*}\right)\right\|_{\beta}^{\beta} \\
\leq & E\left(t^{*}\right) \leq E(0)<d .
\end{aligned}
$$

So, the case $J\left(u\left(t^{*}\right)\right)=d$ is impossible.

Assume that $K\left(u\left(t^{*}\right)\right)=0$ holds, then we get that

$$
\frac{d}{d \lambda} J\left(\lambda u\left(t^{*}\right)\right)=m_{0} \lambda\left(1-\lambda^{\beta-2}\right)\|\nabla u\|^{2} .
$$

We obtain from $\frac{d}{d \lambda} J\left(\lambda u\left(t^{*}\right)\right)=0$ that $\lambda=1$.

Since

$$
\left.\frac{d^{2}}{d \lambda^{2}} J\left(\lambda u\left(t^{*}\right)\right)\right|_{\lambda=1}=-m_{0}(\beta-2)\left\|\nabla u\left(t^{*}\right)\right\|<0 .
$$

Consequently, we get from (2.5) that

$$
\sup _{\lambda \geq 0} J\left(\lambda u\left(t^{*}\right)\right)=\left.J\left(\lambda u\left(t^{*}\right)\right)\right|_{\lambda=1}=J\left(u\left(t^{*}\right)\right)<d
$$

which contradicts the definition of $d$. Therefore, the case $K(u(t))=0$ is impossible as well. Thus, we conclude that $u(t) \in S$ on $[0, T)$.

\section{Main Results and Proof}

Theorem 3.1 Suppose that (2.2) holds, and $u(t)$ is a local solution of problem (1.1)-(1.3) on $[0, T)$. If $u_{0} \in S, u_{1} \in L^{2}(\Omega)$ and $E(0)<d$, then $u(x, t)$ is a global solution of the problem (1.1)-(1.3).

Proof It suffices to show that $\|\nabla u(t)\|^{2}+\left\|u_{t}(t)\right\|^{2}$ is bounded independently of $t$.

Under the hypotheses in Theorem 3.1, we get from Lemma 2.5 that $u(t) \in S$ on $[0, T)$. So the following formula holds on $[0, T)$.

$$
\begin{gathered}
J(u(t))=\frac{m_{0}}{2}\|\nabla u(t)\|^{2}-\frac{b}{\beta}\|u(t)\|_{\beta}^{\beta} \\
\geq \frac{m_{0}}{2}\|\nabla u(t)\|^{2}-\frac{b}{\beta}\|\nabla u(t)\|^{2} \\
=\frac{(\beta-2) m_{0}}{2 \beta}\|\nabla u(t)\|^{2}
\end{gathered}
$$

Therefore, we have from (3.1) that

$$
\begin{aligned}
& \frac{1}{2}\left\|u_{t}(t)\right\|^{2}+\frac{(\beta-2) m_{0}}{2 \beta}\|\nabla u(t)\|^{2} \\
& \leq \frac{1}{2}\left\|u_{t}(t)\right\|^{2}+J(u(t))=E(t) \leq E(0)<d .
\end{aligned}
$$

Hence, we get

$$
\left\|u_{t}(t)\right\|^{2}+\|\nabla u(t)\|^{2} \leq \max \left(2, \frac{2 \beta}{(\beta-2) m_{0}}\right) d<+\infty .
$$

The above inequality and the continuation principle lead to the existence of global solution, that is, $T=+\infty$. Therefore, the solution $u(t)$ is a global solution of the problem (1.1)-(1.3).

The following Theorem shows the exponential decay estimate of global solutions for problem (1.1)-(1.3).

Theorem 3.2 If the hypotheses in Theorem 3.1 are valid, then the global solutions of problem (1.1)-(1.3) has the following exponential decay property

$$
E(t) \leq E(0) e^{1-\frac{t}{M}}
$$

where $M>0$ is a constant.

Proof Multiplying by $u$ on both sides of the Equation (1.1) and integrating over $\Omega \times[0, T)$, we obtain that 
$0=\int_{S}^{T} \int_{\Omega} u\left[u_{t t}-\varphi\left(\|\nabla u\|_{2}^{2}\right) \Delta u-a \Delta u_{t}-b|u|^{\beta-2} u\right] d x d t$,

where $0 \leq S<T<+\infty$.

Since

$$
\int_{S}^{T} \int_{\Omega} u u_{t t} d x d t=\left.\int_{\Omega} u u_{t} d x\right|_{S} ^{T}-\int_{S}^{T} \int_{\Omega}\left|u_{t}\right|^{2} d x d t .
$$

So, substituting the Formula (3.4) into the right-hand side of (3.3), we get that

$$
\begin{aligned}
0 & =\int_{S}^{T}\left(\left\|u_{t}\right\|^{2}+\varphi\left(\|\nabla u\|_{2}^{2}\right)\|\nabla u\|^{2}-\frac{2 b}{\beta}\|u\|_{\beta}^{\beta}\right) d t \\
& -\int_{S}^{T} \int_{\Omega}\left[2\left|u_{t}\right|^{2}-a \nabla u_{t} \nabla u\right] d x d t \\
& +\left.\int_{\Omega} u u_{t} d x\right|_{S} ^{T}+\left(\frac{2}{\beta}-1\right) b \int_{S}^{T}\|u\|_{\beta}^{\beta} d t .
\end{aligned}
$$

It follows from (3.2) that

$\|\nabla u(t)\|^{2} \leq \frac{2 \beta}{(\beta-2) m_{0}} E(t) \leq \frac{2 \beta}{(\beta-2) m_{0}} E(0)<\frac{2 \beta}{(\beta-2) m_{0}} d$

By exploiting Lemma 2.1 and (3.6), we easily arrive at

$$
\begin{aligned}
b\|u\|_{\beta}^{\beta} \leq & b C^{\beta}\|\nabla u(t)\|^{\beta}=b C^{\beta}\|\nabla u(t)\|^{\beta-2}\|\nabla u(t)\|^{2} \\
& <b C^{\beta}\left(\frac{2 \beta}{(\beta-2) m_{0}} d\right)^{\frac{\beta-2}{2}}\|\nabla u(t)\|^{2},
\end{aligned}
$$

We obtain from (3.6) and (3.7) that

$$
\begin{aligned}
b\left(1-\frac{2}{\beta}\right) & \|u\|_{\beta}^{\beta} \leq b C^{\beta}\left(\frac{2 \beta}{(\beta-2) m_{0}} d\right)^{\frac{\beta-2}{2}} \frac{\beta-2}{\beta}\|\nabla u(t)\|^{2} \\
& \leq b C^{\beta}\left(\frac{2 \beta}{(\beta-2) m_{0}} d\right)^{\frac{\beta-2}{2}} \frac{\beta-2}{\beta} \cdot \frac{2 \beta}{(\beta-2) m_{0}} E(t) \\
& =\frac{2 b C^{\beta}}{m_{0}}\left(\frac{2 \beta}{(\beta-2) m_{0}} d\right)^{\frac{\beta-2}{2}} E(t) .
\end{aligned}
$$

We derive from (1.4) that

$$
\int_{0}^{\|\nabla u\|^{2}} \varphi(s) d s \leq \varphi\left(\|\nabla u\|^{2}\right)\|\nabla u\|^{2},
$$

It follows from (3.5), (3.8) and (3.9) that

$$
\begin{aligned}
& 2\left[1-\frac{b C^{\beta}}{m_{0}}\left(\frac{2 \beta}{(\beta-2) m_{0}} d\right)^{\frac{\beta-2}{2}}\right] \int_{S}^{T} E(t) d t \\
& \leq \int_{S}^{T} \int_{\Omega}\left[2\left|u_{t}\right|^{2}-a \nabla u_{t} \nabla u\right] d x d t-\left.\int_{\Omega} u u_{t} d x\right|_{S} ^{T} .
\end{aligned}
$$

We have from Lemma 2.1 and (3.2) that

$$
\begin{aligned}
& \left|\int_{\Omega} u u_{t} d x\right|_{S}^{T}\left|\leq\left(\frac{1}{2}\|u\|^{2}+\frac{1}{2}\left\|u_{t}\right\|^{2}\right)\right|_{S}^{T} \\
& \leq\left.\left(\frac{\beta C^{2}}{(\beta-2) m_{0}} \cdot \frac{(\beta-2) m_{0}}{2 \beta}\|\nabla u\|^{2}+\frac{1}{2}\left\|u_{t}\right\|^{2}\right)\right|_{S} ^{T} \\
& \leq\left.\max \left(\frac{\beta C^{2}}{(\beta-2) m_{0}}, 1\right) E(t)\right|_{S} ^{T} \leq M E(S),
\end{aligned}
$$

Substituting the estimate (3.11) into (3.10), we conclude that

$$
\begin{aligned}
& 2\left[1-\frac{2 b C^{\beta}}{m_{0}}\left(\frac{2 \beta}{(\beta-2) m_{0}} d\right)^{\frac{\beta-2}{2}}\right] \int_{S}^{T} E(t) d t \\
& \leq \int_{S}^{T} \int_{\Omega}\left[2\left|u_{t}\right|^{2}-a \nabla u_{t} \nabla u\right] d x d t+M E(S) .
\end{aligned}
$$

We get from Lemma 2.1 and Lemma 2.3 that

$$
\begin{aligned}
& 2 \int_{S}^{T} \int_{\Omega}\left|u_{t}\right|^{2} d x d t=2 \int_{S}^{T}\left\|u_{t}\right\|^{2} d t \leq 2 C^{2} \int_{S}^{T}\left\|\nabla u_{t}\right\|^{2} d t \\
& =-\frac{2 C^{2}}{a}(E(T)-E(S)) \leq \frac{2 C^{2}}{a} E(S) .
\end{aligned}
$$

From Young inequality, Lemma 2.1, Lemma 2.3 and (3.6), We receive that

$$
\begin{aligned}
& -a \int_{S}^{T} \int_{\Omega} \nabla u_{t} \nabla u d x d t \leq a \int_{S}^{T}\left(\varepsilon\|\nabla u\|^{2}+M(\varepsilon)\left\|\nabla u_{t}\right\|^{2}\right) d t \\
& \leq \frac{2 a \beta \varepsilon}{(\beta-2) m_{0}} \int_{S}^{T} E(t) d t+M(\varepsilon)(E(S)-E(T)) \\
& \leq \frac{2 a \beta \varepsilon}{(\beta-2) m_{0}} \int_{S}^{T} E(t) d t+M(\varepsilon) E(S) .
\end{aligned}
$$

Choosing small enough $\varepsilon$ such that

$$
\frac{2 a \beta \varepsilon}{(\beta-2) m_{0}}+\frac{b C^{\beta}}{m_{0}}\left(\frac{2 \beta}{(\beta-2) m_{0}} d\right)^{\frac{\beta-2}{2}}<1,
$$

then, substituting (3.13) and (3.14) into (3.12),

$$
\int_{S}^{T} E(t) d t \leq M E(S) .
$$

Let $T \rightarrow+\infty$, then we have from (3.15) that

$$
\int_{S}^{+\infty} E(t) d t \leq M E(S) .
$$

Thus, we receive from (3.16) and Lemma 3.1 that

$$
E(t) \leq E(0) e^{1-\frac{t}{M}}, t \in[0,+\infty) .
$$

The proof of Theorem 3.2 is finished.

\section{Acknowledgments}

This Research was supported by Natural Science Foun- 
dation of Zhejiang Province (No.Y6100016), The Science and Research Project of Zhejiang Province Education Commission (No. Y200803804 and Y200907298), The Research Fundation of Zhejiang University of Science and Technology (No. 200803) and the Middleaged and Young Leader in Zhejiang University of Science and Technology (2008-2012).

\section{References}

[1] K. Narasimha, "Nonlinear Vibration of an Elastic String," Journal of Sound and Vibration, Vol. 8, No. 1, 1968, pp. 134-146.

[2] K. Nishihara and Y. Yamada, "On Global Solutions of Some Degenerate Quasilinear Hyperbolic Equations with Dissipative Terms,” Funkcialaj Ekvacioj, Vol. 33, No. 1, 1990, pp. 151-159.

[3] M. Aassila and A. Benaissa, "Existence Globale et Comportement Asymptotique des Solutions des Equations de Kirchhoff Moyennement Degenerees avce un Terme Nonlinear Dissipatif," Funkcialaj Ekvacioj, Vol. 43, No. 2, 2000, pp. 309-333.

[4] K. Ono and K. Nishihara, "On a Nonlinear Degenerate Integro-Differential Equation of Hyperbolic Type with a Strong Dissipation,” Advances in Mathematics Seciences and Applications, Vol. 5, No. 2, 1995, pp. 457-476.
[5] K. Ono, “Global Existence, Decay and Blowup of Solutions for Some Mildly Degenerate Nonlinear Kirchhoff Strings,” Journal of Differential Equations, Vol. 137, No. 1, 1997, pp. 273-301.

[6] P. D. Ancona and S. Spagnolo, "Nonlinear Perturbations of the Kirchhoff Equation," Commnicathins on Pure and Applied Mathematics, Vol. 47, No. 7, 1994, pp. 1005-1029.

[7] M. Ghisi and M. Gobbino, "Global Existence for a Mildly Degenerate Dissipativehyperbolic Equation of Kirchhoff Type,” Preprint, Dipartimento di Matematica Universita di Pisa, Pisa, 1997.

[8] M. Hosoya and Y. Yamada, "On Some Nonlinear Wave Equations II: Global Existence and Energy Decay of Solutions," Journal of the Faculty of Science, The University of Tokyo, Section IA, Mathematics, Vol. 38, No. 1, 1991, pp. 239-250.

[9] L. E. Payne and D. H. Sattinger, "Saddle Points and Instability of Nonlinear Hyperbolic Equations," Israel Jounal of Mathematics, Vol. 22, No. 3-4, 1975, pp. 273-303.

[10] D. H. Sattinger, "On Global Solutions of Nonlinear Hyperbolic Equations,” Archive for Rational Mechanics Analysis, Vol. 30, No. 2, 1968, pp. 148-172.

[11] V. Komornik, "Exact Controllability and Stabilization, The Multiplier Method, RAM: Research in Applied Mathematics,” Masson-John, Wiley, Paris, 1994. 